\title{
FAIR AND STANDARD ACCESS TO SPATIAL DATA AS THE MEANS FOR ACHIEVING SUSTAINABLE DEVELOPMENT GOALS
}

\author{
I. Ivánová ${ }^{1 *}$, N. Brown ${ }^{2}$, R. Fraser ${ }^{3}$, N. Tengku ${ }^{3}$, E. Rubinov $^{4}$, \\ ${ }^{1}$ Spatial Sciences, School of Earth and Planetary Science, Curtin University, Perth, Australia - ivana.ivanova@curtin.edu.au \\ ${ }^{2}$ National Geodesy Section, Geoscience Australia, Canberra, Australia - nicholas.brown@ga.gov.au \\ ${ }^{3}$ Department of Water, Land, Environment and Planning, Melbourne, Australia - (roger.fraser, nuddin.tengku)@dewlp.vic.gov.au \\ ${ }^{4}$ FrontierSI, Melbourne, Australia - erubinov@frontiersi.com.au
}

Commission IV, ICWG IV/III, WG IV/4

KEY WORDS: FAIR, spatial data, standards, interoperability, metadata

\begin{abstract}
:
FAIR, which stands for Findable, Accessible, Interoperable and Reusable, are the main principles adopted for sharing scientific data across communities. Implementing FAIR principles in publishing increases the value of digital resources, and the reuse of these by humans as well as machines. Introducing FAIR practices to the geospatial domain is especially relevant for the foundation geospatial data, such as precise positioning data. Within the next five years, Global Navigation Satellite Systems (GNSS), with corrections from internet or satellite communications, will permit national coverage of positioning services with real-time accuracy of several centimetres or better. However, implementing FAIR principles is not yet common practice in the geospatial domain. There are dozens of standards available for defining and sharing geospatial data. These include the ISO 19100 series of standards, OGC specifications and several community profiles and best practice. However, in most cases these standards fall short in ensuring the FAIR distribution of geospatial resources. As our preliminary findings show, current geodetic metadata and data are not yet fully FAIR and data discovery and access is still very challenging. In this paper we discuss the concept of FAIR and its meaning for geodetic data, explore the needs of precise positioning users and their requirement for metadata and present preliminary results on the FAIRness of current geodetic standards.
\end{abstract}

\section{INTRODUCTION}

Within the next five years, Global Navigation Satellite Systems (GNSS), with corrections from internet or satellite communications, will permit national coverage of positioning services with real-time accuracy of several centimetres or better. This will open up a wide range of positioning applications for new industries (e.g. intelligent transport systems, precision agriculture, location based services etc.) and enable existing industries to improve productivity, efficiency, safety and understanding of the Earth to assist in making important decisions.

Current standards for delivering geodetic data do not adequately serve the needs of new (non-geodetic) users of precise positioning services. Moreover, to improve the efficiency, robustness and accuracy with which data and metadata are shared, there is a need to move away from ASCII flat files targeted to human users predominantly, to machine-to-machine communication. Broad, multi-domain, standards are important for combining geodetic data with data from other domains. Internationally, several groups are working on defining standards for geospatial and geophysical metadata and enhancing interoperability. However, there is no international strategy to ensure geodetic data can be easily discovered, accessed, shared and combined with other data to improve access and maximise data potential. To support new and existing users and to maximise data potential, it is essential that geodetic data is Findable, Accessible, Interoperable and Reusable (FAIR).

FAIR principles need to be adopted for sharing (geo)scientific data across community. The four foundational principles of FAIR (Wilkinson et al., 2016) are aimed at guiding producers and publishers to improve the sustainable use of digital resources (e.g. data, software, services). Implementing FAIR principles in publishing increases the value of digital resources, and the reuse of these by humans as well as machines. Sharing data, information and services in a FAIR way is a practice that will fill critical knowledge gaps required to address the 2030 Agenda for Sustainable Development Goals (SDGs) adopted by the United Nations in 2015 (UN, 2015).

In this paper, we discuss the fundamentals of FAIR principles and their importance for geospatial community. We summarize crucial metadata requirements of precise positioning users in expected high use sectors (e.g. precision agriculture, intelligent transport, marine, aviation, rail and road transportation, and smart location based services) together with our preliminary findings about the gaps to ensure FAIR access to geospatial data as observed from the review of selected existing standards for geographic information.

\footnotetext{
* Corresponding author
} 


\section{BACKGROUND}

\subsection{What is FAIR and why does it matter?}

FAIR principles are the four foundational principles aimed at guiding producers and publishers to improve the sustainable use of digital resources such as data, software or services (Wilkinson et al., 2016). The FAIR guiding principles originate in 2007, when the Organization for Economic Co-operation and Development (OECD) published their principles and guidelines for access to publicly funded research data (OECD, 2007). Currently several funder data policies exist (Hodgson and Molloy, 2015) to ensure intelligently open, accessible and reusable publicly funded research (The Royal Society, 2012).

Implementing FAIR principles in publishing increase the value of digital resources and their reuse by humans as well as machines. FAIR principles apply to digital resources regardless of their public availability and do not require these resources to be open (EC, 2018). However, as indicated as best practice in Open Science, FAIR and Open should be considered as complementary by data practitioners, and resources created from public funds need to be as open as possible and only as closed as necessary (EC, 2018). Several organizations and communities exist to promote FAIR practices including Go FAIR $^{1}{ }$ CODATA $^{2}$ and the Research Data Alliance ${ }^{3}$ ). Geoscience community (e.g. Earth Science Information Partnership and its Australian counterpart Earth and Environment Science Information Partnership) champions the FAIR cause by creating FAIR data repositories (e.g. Australian Ocean Data Network Portal ${ }^{4}$ dedicated to register marine and climate scientific data, or a general resource like Figshare ${ }^{5}$ ). It is also involved in upskilling scientists in FAIR practice (e.g. webinars on FAIR at DataONE ${ }^{6}$ ). Several scientific journals accept supplementary material related to their publications only when these are submitted to a FAIR data repository (Stahl et al., 2019).

\subsection{The FAIR guiding principles}

According to Wilkinson et al. (2016), resources are FAIR when they are findable, accessible, interoperable and reusable. More specifically, the resources are:

Findable when:

F1. (meta)data are assigned a globally unique and persistent identifier

F2. data are described with rich metadata (defined by the R1 below)

F3. metadata clearly and explicitly include the identifier of the data it describes

F4. (meta)data are registered or indexed in searchable resource

Accessible when:

A1. (meta)data are retrievable by their identifier using a standardized communications protocol

\footnotetext{
${ }^{1}$ https://www.go-fair.org/

${ }^{2} \mathrm{http}: / /$ www.codata.org/

${ }^{3} \mathrm{https}: / /$ www.rd-alliance.org/

${ }^{4} \mathrm{https}$ ://portal.aodn.org.au/

${ }^{5} \mathrm{https}: / /$ figshare.com/

${ }^{6} \mathrm{https}: / /$ www.dataone.org/webinars/enabling-fair-data/ and https://www.dataone.org/webinars/quantifying-fair-
}

A1.1. the protocol is free, open and universally implementable

A1.2. the protocol allows for an authentication and authorisation procedure, where necessary

A2. metadata are accessible, even when the data are no longer available

Interoperable

I1. (meta)data use a formal, accessible, shared, and broadly applicable language for knowledge representation

I2. (meta)data uses vocabularies that follow FAIR principles

I3. (meta)data include qualified references to other (meta)data

Reusable

R1. (meta)data are richly described with a plurality of accurate and relevant attributes

R1.1. (meta)data are released with a clear and accessible data usage license

R1.2. (meta)data are associated with data provenance

R1.3 (meta)data meet domain relevant community standards

In more detail, data are findable when they are sufficiently described by their metadata and, when they are registered and indexed in a searchable resource that is known and accessible to potential users (EC, 2018; Wilkinson et al., 2016). For example, resources registered in Geoscience Australia's data and product catalogue can be considered findable because they comply with the requirements F1-F4 described above to large extent.

Digital resources are accessible, when anyone (human or machine) with access to the Internet understands via provided metadata exactly how it is possible to access the digital resource and what are the conditions on its reuse (EC, 2018 Wilkinson et al., 2016). Common misinterpretation of this concept is the expectation that accessible (and hence FAIR) digital objects should be 'open' and/or 'free'. This is not what FAIR guiding principles define. The only condition for FAIR digital objects is the clarity and transparency on the conditions of access and reuse of these objects (Mons et al., 2017). For example, resources registered in Geoscience Australia (GA)'s data and product catalogue can be considered accessible because they comply with the requirements A1-A2 described above to large extent.

Referring to the semantic interoperability of digital resources, these are interoperable when they use a "normative and community recognised specifications, vocabularies and standards that determine the precise meaning of concepts and qualities that the data represent" (EC, 2018, p.19). Although presence of these vocabularies and standards in a format compatible with semantic web would undoubtedly increase their interoperability (van der Brink et al., 2019; Mons et al., 2017), this requirement does not mean that vocabularies and standards used to describe the resource have to 'be on the web'. Use of a well-defined community profile (e.g. GA Metadata Profile Extension for ISO 19115-1:2014, version 2.0) and providing metadata in a machine-readable format (e.g. XML) ensures sufficient interoperability of a resource (e.g. those resources registered in GA's data and product catalogue).

metadata-improvement-and-guidance-dataone-repositorynetwork/ 
License information and the description of the provenance are the two crucial factors determining the reuse of a digital resource (EC, 2018). In addition, both humans and machines, should be able to reuse the digital resources (Mons et al., 2017), which requires the description of the license and provenance information to be provided in a suitable format (e.g. XML or RDF). Resources in GA's data and product catalogue comply with these principles only partially - although both, the license information and the provenance information is provided, it is available only as free-text and this would impede full 'understanding' of these by a machine.

\subsection{FAIR Digital Objects and FAIR ecosystem}

FAIR principles do not just apply to data, but to all digital objects. FAIR Digital Objects represent data, software, protocols and other resources with following characteristics:

- $\quad$ They are accompanied by persistent identifiers (PIDs) and metadata rich enough to enable them to be found, used and cited;

- They are represented in common, ideally open, format and using vocabularies adopted by the community (to enable interoperability); and

- They explain dependencies and licensing.

FAIR ecosystem comprises services and infrastructure for FAIR and contain following components:

- Policies,

- Data management plans,

- Identifiers,

- Standards (including standards for vocabularies, data access and exchange), and

- $\quad$ Repositories (ideally certified with CoreTrustSeal ${ }^{7}$; example of CoreTrustSeal repository is CSIRO's Data Access Portal ${ }^{8}$ )

Implementing FAIR Digital Objects and developing a FAIR ecosystem requires two high-priority activities (EC, 2018):

1. Development, refinement and adoption of shared vocabularies, ontologies, metadata specifications and standards central to interoperability and reuse of FAIR Digital Objects, and

2. Increased provision and professionalization of data stewardship, data repositories and data services.

\subsection{FAIR is a scale}

Implementation of FAIR varies by community, which will decide on which data should be FAIR and to what degree. In this sense, FAIR needs to be understood as scale and various degrees of FAIRness for different types of digital objects are hence possible. One example is illustrated in Figure 1, which shows the variability in FAIR score across more than 600,000 metadata records stored in repositories of the Data Observation Network for Earth (DataONE). These are preliminary results as presented by Jones and Slaughter in 14 May 2019 (Jones and Slaughter, 2019). In the example in Figure 1, we can observe that most DataONE resources are findable and accessible (i.e. comply with most ' $F$ ' and ' $A$ ' requirements as defined in Section 1.2), and, with only few extremes, their FAIRness

\footnotetext{
${ }^{7}$ https://www.coretrustseal.org/

${ }^{8} \mathrm{https}: / /$ data.csiro.au/collections/
}

compliance scores mostly vary between 75 and 100 per cent. However, resources' interoperability and reusability scores are significantly lower. The reasons for this difference are currently being investigated with more comprehensive test, but first investigation suggests that low scores can be explained by incomplete essential metadata and/or missing FAIR checks (Jones and Slaughter, 2019).

\section{Data@NE}

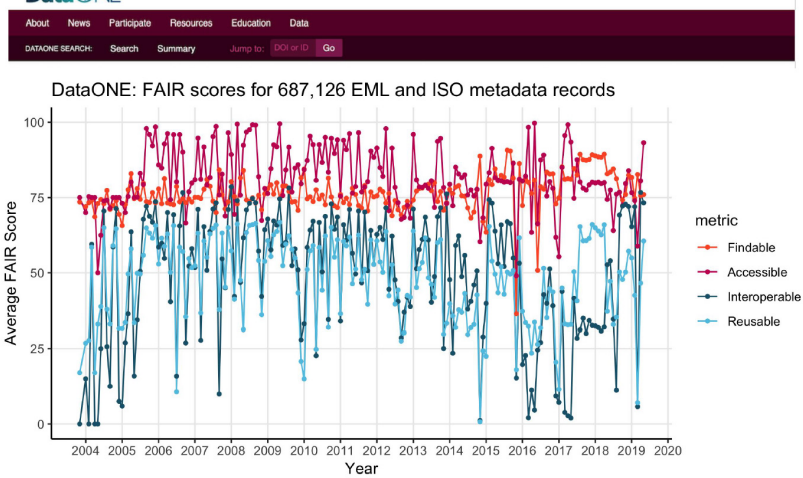

Figure 1 Example of monthly average FAIRness score variability across metadata records (Jones and Slaughter, 2019)

In another investigation Mons et al. (2017) argue that despite digital resources being increasingly FAIR, still more than $80 \%$ of the datasets in current practice are re-useless. This is the term to associate with low FAIR score. Re-useless data include datasets with unknown or unclear provenance and published on obscure or unstable links, not having machine-resolvable persistent and unique identifier (Mons et al., 2017).

\section{METADATA AS THE CRUCIAL ELEMENT FOR ENSURING FAIRNESS}

Metadata are crucial for ensuring FAIRness of digital resources. Metadata can be intrinsic and user-defined (Mons et al., 2017). Intrinsic metadata are created automatically during data capture and user-defined metadata are added to provide context for understanding digital object's creation (e.g. through provenance information). Both types of metadata should be added to a digital resource to ensure its FAIRness.

In geospatial domain, the main digital exchange resource are metadata. These are often maintained separately from the resource itself. Spatial Data Infrastructure (SDI) provides indirect access to the resource via its metadata catalogue. Ideally SDIs should provide metadata records containing links to any related resources, but in reality, this process is not as smooth and typically 'happens' in at least three steps: 1) users (human or machine) access SDI catalogue and retrieve metadata of interest, 2) parse the metadata and compare values in crucial fields (e.g. spatial and temporal extent, time of last update, lineage) with acceptable values for these, and, once the resource fit for intended use is identified, 3) follow the link to this resource. Links to the resource are not necessarily online web links.

For example, a digital resource offered through Data and Publication portal of Geoscience Australia called 'Geodesy Continuously Operating' advertises "Data collected from the Australian Regional Global Navigation Satellite System (GNSS) network, AuScope network and other GNSS observatories located around the world over the last 15 years." 9 According to

\footnotetext{
${ }^{9} \mathrm{http} / / /$ pid.geoscience.gov.au/dataset/ga/74501
} 
the corporate metadata specification (GA, 2014) as illustrated in Figure 2, one would expect links to a resource this metadata record describes (e.g. data and site logs from GNSS observatories) in the metadata file upon opening the metadata file of this product.

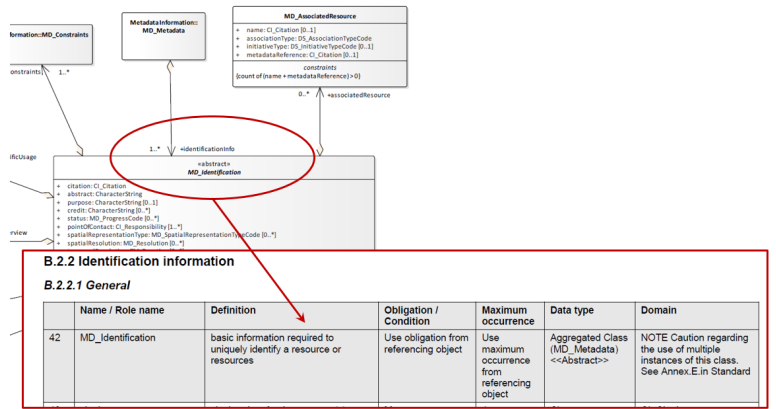

Figure 2 Resource identification information and related obligation as defined in (GA, 2014)

Unfortunately, as illustrated in Figure 3, this is not the case the reference to resources related 'Geodesy - Continuously Operating' product is not present its corporate standard compliant (GA, 2014) metadata.

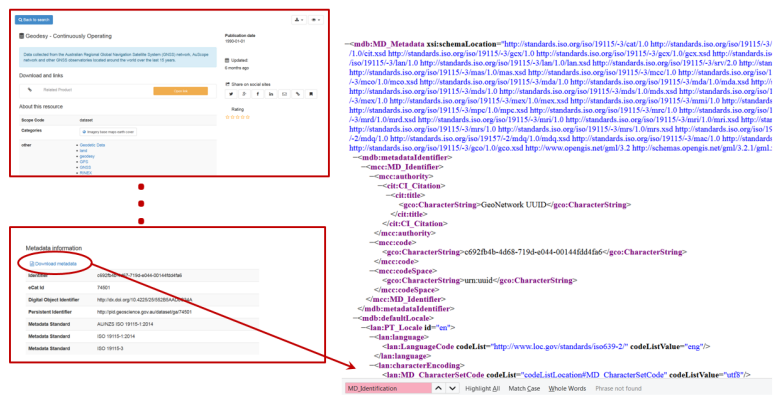

Figure 3 Missing identification of resources related to 'Geodesy - Continuously Operating' product.

However, on the landing information page for human access to the catalogue (Figure 4), there is a link to a location which holds the associated resource to the product.

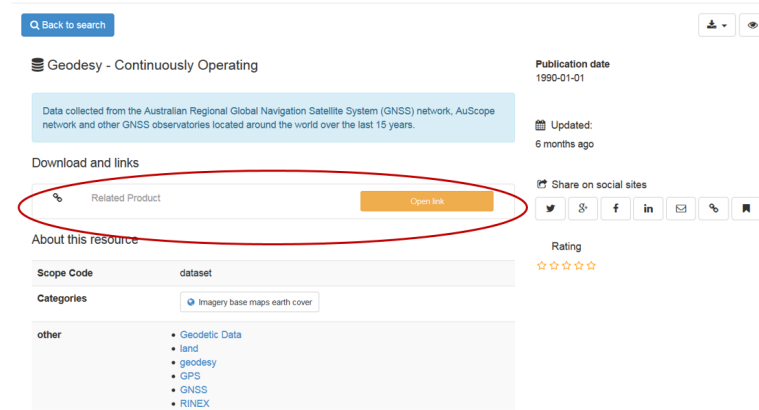

Figure 4 Landing page for human metadata consumption with link to related products to 'Geodesy - Continuously Operating'

But again, this link takes several additional manual clicks to get to the resource as illustrated in Figure 5.

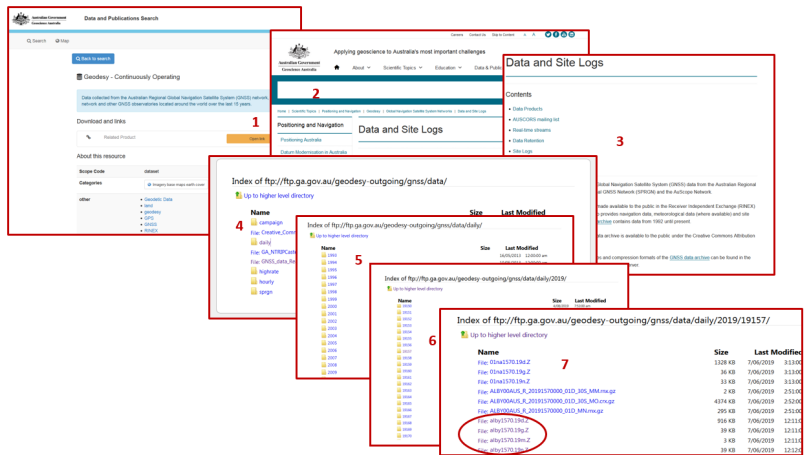

Figure 5 Navigating to the digital resource of interest advertised by the metadata of 'Geodesy - Continuously Operating'

This is all very well manageable by an educated searcher (e.g. a GNSS specialist), but it's hard to imagine that without precisely crafted procedure (again, by a human), a machine would be capable to find a resource, and hence impede FAIR access to precise positioning users.

\section{USERS OF PRECISE POSITIONING DATA AND THEIR REQUIREMENTS ON METADATA}

As identified in the recent report on the benefits of satellitebased augmentation system (SBAS), the following are the current high-end user sectors for precise positioning data (Teunissen and Montembruck, 2017; Ernst Young, 2019):

- $\quad$ Agriculture - precision machinery monitoring, spray applications, fertilising, top dressing and yield mapping

- $\quad$ Rail - management systems, rail line mapping and integrity monitoring, vehicle training

Road - connected and automated vehicle (CAV) tracking, asset and road furniture management, traffic flow monitoring, tolling (offroad), truck/fleet management, and advanced driver advisory systems

- $\quad$ Maritime - boat tracking, navigation, underkeel clearance, cable protection zones and exclusion areas, and compliance of fisheries and parks

- $\quad$ Aviation - drone applications, precision landing and navigation for aircraft landing, data (e.g. LiDAR) acquisition

Intelligent transportation \& Location Based Services - underground services, people movement and pedestrian navigation, enhanced search and rescue, and mapping, mobile workforce management and tracking, sport and well-being tracking, personal use mapping, mobile integration (e.g. ride-sharing applications)

- $\quad$ Surveying \& Resources - Building Information Models (BIM), precision guidance, drone application, resource supply chain, exploration and mine operation tracking

Utilities \& Time synchronisation - drone asset management and inspection, electrical network synchronisation

Table 1 shows the summary of user requirements for GNSS metadata (Teunissen and Montembruck, 2017; GSAa, 2019; GSAb, 2019). Metadata elements displayed in Table 1 enable users on the decision of precise positioning data fitness for intended purpose within the sector. 
Table 1 Users and their metadata requirements on precise positioning data

\begin{tabular}{|c|c|}
\hline User sector & Metadata requirements \\
\hline Agriculture & $\begin{array}{l}\text { Accuracy } \\
\text { Service area/coverage } \\
\text { Availability } \\
\text { Integrity } \\
\text { Robustness } \\
\text { Relevance of the surveying method } \\
\text { Time To First Fix (TTFF) } \\
\text { Size, weight and autonomy/Power } \\
\text { consumption } \\
\text { Interoperability and software flexibility } \\
\text { Real-time and post-processed capability } \\
\text { Multi-functionality and compatibility } \\
\text { with other sensors (e.g. bar code readers) } \\
\text { Continuity } \\
\text { Antenna ruggedness, performance and } \\
\text { phase-centrum stability } \\
\text { Time To Convergence (TTC) } \\
\text { Reliability }\end{array}$ \\
\hline Rail & $\begin{array}{l}\text { Accuracy of location } \\
\text { Time stamp of location measurement } \\
\text { Accuracy of speed } \\
\text { Availability } \\
\text { Safe clock drift } \\
\text { Integrity } \\
\text { Safety integrity Level } \\
\text { Time To Alert (TTA) } \\
\text { Reliability measured in Mean Time } \\
\text { Between Failures (MTBF) }\end{array}$ \\
\hline Road & $\begin{array}{l}\text { Availability } \\
\text { Accuracy } \\
\text { GNSS Time accuracy } \\
\text { Integrity message } \\
\text { TTFF } \\
\text { Robustness to interference: jamming, } \\
\text { spoofing, multipath } \\
\text { Authenticity, incl. provision of the } \\
\text { authentication message ensuring users } \\
\text { that the signal comes from a valid source } \\
\text { (enabling sensitive applications) } \\
\text { Detection of GNSS interferences } \\
\text { GNSS sensitivity } \\
\text { Continuity (SM) } \\
\text { Position fix rate } \\
\text { Latency } \\
\text { Indoor penetration } \\
\text { Power consumption indication }\end{array}$ \\
\hline Maritime & $\begin{array}{l}\text { Accuracy; max error } \\
\text { Availability } \\
\text { Continuity } \\
\text { Integrity: Warning, Risk, Alert limit, } \\
\text { TTA } \\
\text { Coverage } \\
\text { Horizontal alert limit } \\
\text { TTFF } \\
\text { Update rate of the computed position } \\
\text { data } \\
\text { Support for International Association of } \\
\text { Lighthouse Authorities (IALA) DGNSS } \\
\text { service and the Automatic Identification } \\
\text { System (AIS) }\end{array}$ \\
\hline
\end{tabular}

\begin{tabular}{|c|c|}
\hline Aviation & $\begin{array}{l}\text { Accuracy } \\
\text { Horizontal/Vertical Alert Limit } \\
\text { Velocity accuracy } \\
\text { Time accuracy } \\
\text { System Design Assurance } \\
\text { Positon integrity } \\
\text { Integrity } \\
\text { TTA } \\
\text { Continuity } \\
\text { Availability }\end{array}$ \\
\hline $\begin{array}{l}\text { Intelligent } \\
\text { transportation \& } \\
\text { Location Based } \\
\text { Services }\end{array}$ & $\begin{array}{l}\text { Accuracy } \\
\text { Authentication } \\
\text { Resilience/Robustness: } \\
\text { Susceptibility to interference and } \\
\text { jamming } \\
\text { Susceptibility to spoofing } \\
\text { Susceptibility to environmental } \\
\text { conditions, incl. multipath effects } \\
\text { Availability } \\
\text { Position, navigation, timing in urban } \\
\text { canyons, under canopy and indoors } \\
\text { TTFF } \\
\text { Fix update type } \\
\text { Power consumption alert } \\
\text { Integrity messages: risk, TTA } \\
\text { Service area/Coverage }\end{array}$ \\
\hline $\begin{array}{l}\text { Surveying } \quad \& \\
\text { Resources }\end{array}$ & $\begin{array}{l}\text { Accuracy } \\
\text { Service area/coverage } \\
\text { Availability } \\
\text { Integrity } \\
\text { Robustness } \\
\text { Relevance of the surveying method } \\
\text { TTFF } \\
\text { Size, weight and autonomy/Power } \\
\text { consumption } \\
\text { Interoperability and software flexibility } \\
\text { Real-time and post-processed capability } \\
\text { Multi-functionality and compatibility } \\
\text { with other sensors (e.g. bar code readers) } \\
\text { Continuity } \\
\text { Antenna ruggedness, performance and } \\
\text { phase-centrum stability } \\
\text { TTC } \\
\text { Reliability }\end{array}$ \\
\hline $\begin{array}{l}\text { Utilities \& Time } \\
\text { synchronisation }\end{array}$ & $\begin{array}{l}\text { Accuracy: positional, temporal } \\
\text { Resilience and reliability } \\
\text { Authentication } \\
\text { Availability } \\
\text { Traceability } \\
\text { Trustability } \\
\text { Security } \\
\text { Integrity } \\
\text { Independence of GNSS Timing \& } \\
\text { Synchronisation System } \\
\text { Certification } \\
\text { Protocols: Network time Protocol and } \\
\text { Precision Time Protocol, IRIG-B, } \\
\text { SyncE, SONET/SDH }\end{array}$ \\
\hline
\end{tabular}

\section{ARE GEODETIC STANDARDS FAIR?}

Implementing FAIR principles is not yet common practice in the geospatial domain. There are dozens of standards available for defining and sharing geospatial data. These include the ISO 
19100 series of standards, OGC specifications and several community profiles and best practice. However, in most cases these standards fall short in ensuring the FAIR distribution of geospatial resources. As our preliminary findings show, data discovery and access is still a very challenging, and at times, close to impossible exercise. There are several reasons for this, and here we mention just three examples: 1) gaps in metadata provided by geospatial data producers and mandated by the community of practice (e.g. within stakeholders of the spatial data infrastructure), 2) non-interoperable data exchange standards containing insufficient metadata, which are declared in a local jargon (e.g. language of geodetic science, which might not be fully comprehensible by the users of other sectors), and 3) metadata provided in a free-form text not linked to any open vocabulary, which makes it impossible for machines to parse and hence use these metadata automatically.

During our preliminary experiments we manually analysed the status of metadata and data records as distributed to their users currently. Table 2 and Table 3 provide an overview FAIRness status of metadata and data record respectively, as measured with selected online FAIRness self-assessment tools.

Table 2 FAIRness status of geodetic metadata record - tested example dataset: Geodesy - Continuously Operating ${ }^{10}$

\begin{tabular}{|l|l|}
\hline Tool & GA record FAIR score \\
\hline $\begin{array}{l}\text { ANDS NECTAR } \\
\text { FAIR Data Self- } \\
\text { assessment Tool }^{11}\end{array}$ & Approx. over 60\% (no numeric result) \\
\hline DANS-Fairdat & \\
\hline $\begin{array}{l}\text { DANS-Fair } \\
\text { enough? }\end{array}$ & $\begin{array}{l}4.67 \text { out of } 5 \text { calculated as }(\mathrm{F}+\mathrm{A}+\mathrm{I}) / 3 ; \\
\text { note: the tool does not test 'R'. }\end{array}$ \\
\hline $\begin{array}{l}\text { The CSIRO 5-star } \\
\text { Data Rating tool }\end{array}$ & $3.48^{14}$ out of $5^{*}$ \\
\hline $\begin{array}{l}\text { FAIR Metrics } \\
\text { Questionnaire }\end{array}$ & $\begin{array}{l}12 \text { pass; } 8 \text { fail; 1 problematic; 1 not } \\
\text { applicable (out of 22 questions in total) }\end{array}$ \\
\hline
\end{tabular}

Table 3 FAIRness status of geodetic data record

\begin{tabular}{|l|l|}
\hline Tool & GA record FAIR score \\
\hline $\begin{array}{l}\text { ANDS NECTAR } \\
\text { FAIR Data Self- } \\
\text { assessment Tool }\end{array}$ & $\begin{array}{l}\text { No numeric result, only colour } \\
\text { indicator showing approx. 25\% }\end{array}$ \\
\hline DANS-Fairdat & $\begin{array}{l}1.67 \text { out of 5 calculated as }(\mathrm{F}+\mathrm{A}+\mathrm{I}) / 3 ; \\
\text { note: the tool does not test 'R'. }\end{array}$ \\
\hline $\begin{array}{l}\text { DANS-Fair } \\
\text { enough? }\end{array}$ & 7 out of 13 points \\
\hline $\begin{array}{l}\text { The CSIRO 5-star } \\
\text { Data Rating tool }\end{array}$ & $0.78^{*}$ out of 5* \\
\hline $\begin{array}{l}\text { FAIR Metrics } \\
\text { Questionnaire }\end{array}$ & $\begin{array}{l}6 \text { pass; 15 fail; 0 problematic; 1 not } \\
\text { applicable (out of 22 questions in total) }\end{array}$ \\
\hline
\end{tabular}

\footnotetext{
${ }^{10} \mathrm{http}: / /$ pid.geoscience.gov.au/dataset/ga/74501

${ }^{11} \mathrm{https}$ ://www.ands-nectar-rds.org.au/fair-tool

${ }^{12} \mathrm{https}: / /$ www.surveymonkey.com/r/fairdat

${ }^{13} \mathrm{https} / / /$ docs.google.com/forms/d/e/1FAIpQLSf7t1Z9IOBoj5G gWqik8KnhtH3B819Ch61D5KuAz7yn0I0Opw/viewform

${ }^{14} \mathrm{http}: / /$ oznome.csiro.au/5star/

${ }^{15} \mathrm{https} / /$ github.com/FAIRMetrics/Metrics/blob/master/Evaluati on_Of_Metrics/Supplementary Information_ FM Evaluation Results.pdf
}

As can be observed from the results in Table 2 and Table 3, the geodetic data are on their way to support FAIR principles. The deficiencies causing low FAIR score are similar as indicated by (Jones and Slaughter, 2019; Mons et al., 2017) - current metadata and data records miss elements of interoperability and reusability, which include machine-readable record of resource's provenance, machine-readable license information, links to well-defined and established domain vocabularies, etc. In addition to the heterogeneity of standards for data exchange, there is terminological heterogeneity within the geodetic community itself. For instance, some geodesists use the term 'GNSS station' whereas others say 'GNSS site' to point out the same location with continuous observations. To reduce such confusions, Geodesy Markup Language (GeodesyML ${ }^{16}$ ) has been proposed as a way towards standardisation of encoding and sharing geodetic data and metadata. In a follow-up research to the presented in this paper is to analyse the FAIR capability of GeodesyML and propose improvements to the next version of this standard.

\section{FAIR GEODETIC DATA AND SDGS}

Improving geodetic standards to ensure FAIR precise positioning data. More specifically, the FAIR precise positioning data ensures timely and accurate access good health and well-being (SDG 3), efficient management of clean water and sanitation (SDG 6) and well-functioning smart and sustainable cities (SDG 11). Moreover, FAIR precise positioning data help in a responsible consumption and production (SDG 12) and assure life below water and on land (SDG 13 and 14). As such, the work presented in this paper aligns with the Agenda 2030 and contributes to the progress in addressing the sustainable development goals as set out by the United Nations (UN, 2015)

\section{CONCLUSION}

In this paper we discussed the need and importance for FAIR precise positioning data as the means to deliver useful information to the current high-end user sectors. We also listed metadata requirements as defined by the users from high-end precise positioning sector as well as provided an overview of the capability of current standards to deliver FAIR access to geodetic data. Our next research includes suggestions for improvement of current geodetic standards with respect to the FAIRness requirements.

\section{ACKNOWLEDGEMENTS}

Research presented in this paper is part of research project on funded and developed collaboratively by Geoscience Australia, Curtin University, Department of Environment, Water, Land and Planning of Victoria, and FrontierSI.

\section{REFERENCES}

Australian National Data Service (ANDS), 2019. FAIR selfassessment tool, URL: https://www.ands-nectar-rds.org.au/fairtool [19 September 2019]

van den Brink, L. Barnaghi, P., Tandy, J., Atemezing, G., Atkinson, R., Cochrane, B., ...Troncy, R. (2019) Best practices for publishing, retrieving and using spatial data on the web,

\footnotetext{
${ }^{16} \mathrm{http}: / /$ geodesyml.org
} 
Semantic Web 10(1), 95-114, http://www.semantic-webjournal.net/system/files/swj1785.pdf [30 September 2019]

Ernst Young, 2019. SBAS Test-bed Demonstrator Trial Economic benefits report, Melbourne: FrontierSI, https://frontiersi.com.au/wp-content/uploads/2018/08/SBASEconomic-Benefits-Report.pdf [11 October 2019]

European Commission (EC), 2018. Turning FAIR into reality Final Report and Action Plan from the European Commission Expert Group on FAIR data, EC: Brussels.

European Global Satellite Agency (GSA), 2019a. EGNOS User satisfaction results, https://egnos-user-support.esspsas.eu/new_egnos_ops/resources-tools/user-satisfaction [11 October 2019]

Geoscience Australia (GA), 2014. GA Metadata Profile Extension for ISO 19115-1:2014, version 2.0, http://pid.geoscience.gov.au/def/schema/ga/ [3 October 2019]

GSA, 2019b. GNSS Users, https://www.gsc-europa.eu/gnssmarket-applications/gnss-users [11 October 2019]

Hodgson, S. and Molloy, L., 2014. Current Best Practice for Research Data Management Policies, ICSU/CODATA, http://doi.org/ 10.5281/zenodo.27872 [14 October 2019]

Jones, M. B. and Slaughter, P., 2019. Quantifying FAIR: metadata improvement and guidance in the DataONE repository network, DataONE webinar presented on 14 May 2019, https://www.dataone.org/webinars/quantifying-fair-metadataimprovement-and-guidance-dataone-repositorynetwork\#abstract [1 October 2019]

Mons, B., Neylon, C., Velterop, J., Dumontier, M., da Silva Santos, L. O. B. and Wilkinson, M. D., 2017. Cloudy, increasigly FAIR; revisiting the FAIR Data guiding princples for the European Open Science Cloud, Information Services \& Use, 37(1), http://doi.org/ 10.3233/ISU-170824 [30 September 2019]

Organization for Economic Co-operation and Development (OECD), 2007. Principles and Guidelines for Access to Research Data from Public Funding, https://doi.org/10.1787/9789264034020-en-fr $\quad[30$ September 2019]

Research Data Alliance (RDA) FAIR Data Maturity Model Working Group, 2019. Results of an analysis of existing FAIR assessment tools, v.0.02, 13 March 2019, https://www.rdalliance.org/system/files/Results\%20initial\%20analysis\%20exis ting\%20FAIR\%20assessment\%20tools\%20v002_0.pdf [2 October 2019]

Stahl, S., Yarmey, L., Cutcher-Gershenfeld, J., Hanson, B., Lehnert, K., Nosek, B., Parsons, M., Robinson, E. and Wyborn, L., 2019. Make scientific data FAIR, Nature 570, $27-29$ (2019), http://doi.org/10.1038/d41586-019-01720-7 [30 September 2019]

The Royal Society, 2012. Science as an Open Enterprise, https://royalsociety.org/-/media/policy/projects/sape/2012-0620-saoe.pdf [30 September 2019]
Teunissen, P. and Montembruck, O. (eds.), 2017 Springer Handbook of GNSS, Berlin: Springer-Verlag, 1327p, doi: 10.1007/978-3-319-42928-1

United Nations (UN), 2015. Transforming our world: The 2030 Agenda for sustainable development, A/RES/70/1, New York: United Nations,

https://sustainabledevelopment.un.org/post2015/transformingou rworld/publication

Wilkinson M.D. et al., 2016. The FAIR Guiding Principles for scientific data management and stewardship. Scientific Data 3:160018, http://doi.org/10.1039/sdata.2016.18 [19 September 2019] 